\title{
Deficiência de ferro no paciente submetido à ressecção gástrica ou intestinal: prevalência, causas, repercussões clínicas, abordagem diagnóstica e prevenção Iron deficiency anemia in patients submitted to gastric or small intestine resection: prevalence, cause, clinical outcome, diagnostic workup and prevention
}

\begin{abstract}
Anemia ferropriva é frequente em pacientes submetidos à ressecção gástrica, para o tratamento de úlcera péptica ou câncer gástrico, e à cirurgia bariátrica, para o tratamento de obesidade. As cirurgias bariátricas podem ter como consequência a restrição do estômago, sem necessariamente ressecção gástrica, ou ressecção gástrica associada ou não a ressecção intestinal. As causas da deficiência de ferro nestes pacientes são multifatoriais e incluem: (1) baixa ingestão de ferro oral por intolerância a alimentos ricos em ferro; (2) redução da acidez gástrica pela perda de células parietais, dificultando a conversão do ferro férrico para a forma ferrosa; (3) exclusão do duodeno com consequente exclusão do principal sítio de absorção do ferro e restrição da ação das enzimas pancreáticas na liberação do ferro ligado ao heme. A investigação e a reposição de ferro de forma eficaz e precoce pode evitar essa complicação. Pacientes refratários ao ferro oral podem necessitar de reposição de ferro parenteral e todos os pacientes requerem acompanhamento periódico com hemograma e estoques de ferro por toda a vida. Rev. Bras. Hematol. Hemoter. 2010; 32(Supl.2):78-83.
\end{abstract}

Palavras-chave: Anemia ferropriva; gastrectomia; cirurgia bariátrica.

\section{Introdução}

Os procedimentos de ressecção gástrica e intestinal são realizados há mais de cem anos. Inicialmente, os procedimentos de gastrectomia a Bilroth I ou Bilroth II eram indicados para o tratamento de úlcera péptica e câncer gástrico. A gastrectomia a Bilroth consiste na retirada de parte do estômago, refazendo o trato gastrintestinal através de anastomose com o duodeno (Bilroth I) ou jejuno (Bilroth II). ${ }^{1}$ Recentemente, a cirurgia bariátrica, procedimento indicado para o tratamento de obesidade mórbida, é a cirurgia de ressecção gástrica associada ou não a ressecção de porções do intestino delgado mais frequentemente realizada. ${ }^{2}$

Obesidade mórbida é um sério problema de saúde pública nos Estados Unidos da América. Em 2002, 5,1\% dos adultos dos EUA apresentavam obesidade mórbida, definida como um índice de massa corpórea acima de $40 \mathrm{~kg} / \mathrm{m}^{2}$. Em 2006, a Sociedade Americana de Cirurgia Bariátrica e Metabólica fez uma estimativa de que 177.600 indivíduos haviam sido submetidos à cirurgia bariátrica nos EUA. ${ }^{3,4}$ No Brasil, dados recentes mostram um aumento de $255 \%$ na prevalência da obesidade mórbida. No período de 1975-1976, apenas $0,18 \%$ da população apresentava os critérios para serem classificados como obesos mórbidos. Essa prevalência passou para $0,64 \%$ no período de 2002-2003. Como consequência, no período entre 1999 a 2006 mais de 10 mil cirurgias bariátricas foram realizadas no Brasil..$^{5}$

A primeira cirurgia bariátrica foi realizada em 1954 por Kremen, que uniu a porção proximal do jejuno ao íleo distal, ignorando um grande segmento do intestino delgado, um

Médica hematologista do Centro de Hematologia e Hemoterapia da Universidade de Campinas (Hemocentro Unicamp) - Campinas-SP.

Centro de Hematologia e Hemoterapia da Universidade Estadual de Campinas (Hemocentro Unicamp) - Campinas-SP.

Correspondência: Fabiola Traina

Rua Carlos Chagas, 480, Cidade Universitária Zeferino Vaz, Barão Geraldo

13083-970 - Campinas-SP - Brasil

Fax: (55 19) 3289-1089

E-mail:fabiolat@unicamp.br

Doi: 10.1590/S1516-84842010005000071 
procedimento denominado bypass jejuno-ileal, resultando em uma alteração na absorção dos alimentos. ${ }^{6}$ Devido à má absorção de carboidratos, lipídeos, vitaminas e proteínas, a cirurgia resultou em graves morbidades, incluindo cirrose hepática e insuficiência hepática em 7\% dos pacientes. ${ }^{7}$ Desde então, dois principais procedimentos de cirurgia bariátrica tornaram-se populares e eficazes em reduzir o peso corpóreo nos casos de obesidade mórbida, procedimentos de banda e bypass gástrico. ${ }^{8}$ A gastroplastia por anel vertical e a banda gástrica ajustável são exemplos de procedimentos de banda. Nesse caso, a perda de peso é induzida pela restrição da ingestão de alimentos. No caso do bypass gástrico, há várias técnicas descritas, como o bypass gástrico em Y de Roux, a derivação biliopancreática, a derivação biliopancreática com derivação duodenal e a Fobicapella. Essas cirurgias induzem a perda de peso através de ressecção gástrica e intestinal com consequente restrição e má absorção dos alimentos. Os resultados mostram que o bypass gástrico é mais eficiente na redução de peso se comparado aos procedimentos de banda. ${ }^{2}$

Na cirurgia de bypass gástrico em Y de Roux é criada uma pequena bolsa gástrica, com $15 \mathrm{~mL}$ a $30 \mathrm{~mL}$ de capacidade, que tem como objetivo limitar de uma forma importante a capacidade de ingestão dos alimentos. Esta bolsa é ligada ao intestino delgado na região do jejuno, ultrapassando assim o duodeno e o jejuno proximal, impedindo a ação das secreções pancreática e biliar. As técnicas de derivação biliopancreática e derivação biliopancreática com derivação duodenal são procedimentos ainda mais radicais, pois excluem uma maior porção do jejuno do trato digestivo. ${ }^{2}$ Uma das técnicas mais utilizadas atualmente é a técnica de Fobi-capella, em que uma pequena bolsa estomacal é criada através do grampeamento vertical. A seguir, uma seção do intestino delgado em forma de "y" com a alça alimentar medindo entre $1,0 \mathrm{~m}$ e 1,5 m é fixada à bolsa para permitir que os alimentos passem ao intestino delgado sem passar no duodeno e primeiras porções do intestino delgado, provocando uma absorção reduzida de calorias e nutrientes. ${ }^{9,10}$

Uma das complicações associadas à gastrectomia a Bilroth I ou Bilroth II e à cirurgia bariátrica é a deficiência de ferro e, consequentemente, a anemia ferropriva. A grande maioria dos estudos descreve a deficiência de ferro variando de $6 \%$ a $50 \%$ dentro de meses a anos de seguimento pósoperatório, sendo que a incidência aumenta a depender do período de seguimento pós-operatório relatado nos estudos. ${ }^{11-13}$ Há relatos de casos de pica, uma condição associada à anemia ferropriva e definida como desejo incomum por gelo, maisena, barro e outras substâncias. ${ }^{14}$

Existem poucos estudos randomizados e controlados que estudam deficiência de ferro em cirurgia bariátrica. Os dados aqui descritos se baseiam em estudos descritivos e retrospectivos. Os exames utilizados para a avaliação do estoque de ferro e o tempo médio de seguimento no pósoperatório variam nos diferentes estudos. A maioria dos pacientes perde o seguimento após dois anos, sendo difícil quantificar a variação laboratorial nesses casos. Além disso, diferentes estoques de ferro no pré-operatório influenciam o tempo de ocorrência de deficiência de ferro e anemia ferropriva, de forma que a incidência destas alterações pode ser subestimada em estudos com curtos períodos de seguimento. $^{15}$

\section{Causas de deficiência de ferro após cirurgia bariátrica}

\section{Intolerância à carne vermelha}

Existem duas formas de ferro disponíveis para absorção: ferro ferroso $\left(\mathrm{Fe}^{2}\right)$ e ferro ligado ao heme. Carne vermelha é a principal fonte de ferro ligado ao heme. ${ }^{16}$ Algumas evidências sugerem que a diminuição na ingestão de carne vermelha após a cirurgia de bypass gástrico contribui para a deficiência de ferro nestes pacientes. É frequente a queixa de náuseas e vômitos após ingestão de carne, e cerca de $50 \%$ dos indivíduos são intolerantes à carne vermelha no pós-operatório. Como consequência, foi observado que os pacientes submetidos à cirurgia bariátrica consomem menos de $70 \%$ das necessidades mínimas de ferro diário, mesmo após vários anos de pós-operatório. ${ }^{17-19}$

A intolerância à carne vermelha é mais frequente nos procedimentos de banda que nos de bypass, apesar da anemia ferropriva ser mais frequente no bypass. A maior intolerância à carne vermelha nos procedimentos de banda justifica-se pelo menor tamanho do reservatório gástrico, e a maior incidência de anemia ferropriva nos procedimentos de bypass indica que a diminuição na ingestão de ferro e carne vermelha não é a única explicação para a deficiência de ferro. ${ }^{20}$

\section{Redução da secreção ácida}

$\mathrm{O}$ metabolismo inicial de ferro férrico $\left(\mathrm{Fe}^{3}\right)$ ocorre no estômago e é facilitado pela acidez do suco gástrico. $\mathrm{O}$ ferro férrico é convertido a ferro ferroso $\left(\mathrm{Fe}^{2}\right)$ no baixo $\mathrm{pH}$ do estômago antes de se tornar disponível para absorção no duodeno alcalino. ${ }^{16}$ Qualquer cirurgia de ressecção gástrica, como Bilroth I e II e bypass gástrico, que envolve ressecção do corpo do estômago, resultará em redução das células parietais e redução na secreção de suco gástrico, impedindo a conversão do ferro férrico para ferro ferroso e reduzindo a absorção do ferro no duodeno. Em contraste, procedimentos de banda gástrica mantêm a comunicação entre o corpo gástrico e o duodeno e as taxas de deficiência nutricional e deficiência de ferro são menores que nos procedimentos de bypass gástrico. ${ }^{21}$

\section{Exclusão do duodeno}

Pacientes submetidos à cirurgia de bypass gástrico, um procedimento que exclui o duodeno da absorção, tem significativamente menor ferro sérico e concentrações de 
hemoglobina que aqueles submetidos a procedimentos de banda, que preservam a integridade duodenal. ${ }^{22} \mathrm{~A}$ quantidade de superfície absortiva do jejuno tem pouco impacto na absorção de ferro, sugerindo que o resto do intestino delgado não pode aumentar a absorção de ferro de forma suficiente para compensar a exclusão duodenal que ocorre em procedimentos de bypass gástrico. Brolin e colaboradores compararam a taxa de deficiência de ferro entre grupos de pacientes submetidos à bypass gástrico que tinham diferentes comprimentos de jejuno funcional e não encontraram diferenças significativas na deficiência de ferro entre os grupos. ${ }^{23} \mathrm{Um}$ estudo prospectivo comparando pacientes com bypass gástrico em Y de Roux e pacientes com derivação biliopancreática encontrou em ambos os grupos níveis de ferritina equivalentes, após dois anos da cirurgia, apesar de diferentes comprimentos de superfície absortiva de jejuno. ${ }^{24}$

O principal local de absorção do ferro ferroso e ferro ligado ao heme é a membrana basolateral e apical dos enterócitos do duodeno. Quando o ferro férrico alcança a borda em escova das células epiteliais do duodeno, o ferro é reduzido da forma férrica para a forma ferrosa pela enzima ferro redutase e transportado através da membrana apical pelo transportador divalente DMT1. Uma vez que o ferro ferroso tenha sido transportado para dentro do enterócito, o ferro é transportado para o plasma através da ferroportina enquanto o ferro em excesso é retido no enterócito como ferritina. ${ }^{25}$ Além da exclusão do duodeno do trânsito intestinal, a cirurgia de bypass gástrico atrasa a interação das enzimas pancreáticas e secreção biliar com o bolo alimentar, diminuindo a habilidade de liberar o heme da mioglobina e hemoglobina. ${ }^{26}$

\section{Outros fatores}

Deficiência de ferro após a cirurgia pode ser devido ao aumento de perda de sangue. Pacientes submetidos a bypass gástrico podem experimentar sangramento gastrintestinal na alça de intestino excluída do trato intestinal. Outras fontes de perda sanguínea incluem úlceras marginais, que sabidamente ocorrem no sítio de anastomose da porção proximal do jejuno e corpo gástrico. Pacientes submetidos a bypass gástrico podem também experimentar uma enteropatia perdedora de ferro e um supercrescimento bacteriano, especialmente na alça cega, resultando em dano e excreção do epitélio intestinal e perda do ferro livre estocado. ${ }^{13}$

Na maioria dos estudos não é possível definir se a rápida perda de peso contribui para a deficiência de ferro. Algumas evidências sugerem que a quantidade de perda de peso e a velocidade com que isso ocorre não têm influência no grau de deficiência de ferro. ${ }^{19}$ Além disso, há poucos estudos descrevendo se os hormônios que têm impacto no metabolismo de ferro, como TSH, T3 e T4, são afetados pela cirurgia. A função tireoidiana anormal tem sido utilizada como critério de exclusão dos estudos, e a avaliação da função tireoidiana não é incluída como rotina da avaliação laboratorial no acompanhamento pós-operatório. ${ }^{27,28}$

\section{Deficiência de ferro e anemia em populações especiais}

\section{Mulheres}

Mulheres em idade menstrual estão em maior risco para deficiência de ferro e anemia ferropriva após cirurgia bariátrica. Baixo estoque de ferro pré-operatório pode parcialmente explicar porque a mulher é mais suscetível a apresentar deficiência de ferro e porque o tempo para ocorrência de deficiência de ferro é tão variado, de meses a anos. O retorno da menstruação no pós-operatório pode contribuir para o aumento das taxas de deficiência de ferro em mulheres. Um estudo avaliando as alterações ginecológicas em 109 mulheres com obesidade mórbida em idade fértil submetidas à cirurgia bariátrica identificou a presença de irregularidade menstrual em $40,4 \%$ das mulheres no pré-operatório e em apenas 4,6\% após a perda de peso, sugerindo que a cirurgia bariátrica pode corrigir o estado anovulatório e de resistência à insulina associada à obesidade e síndrome do ovário policístico. ${ }^{29} \mathrm{~A}$ predisposição de mulheres em idade menstrual de desenvolver anemia ferropriva se mantém mesmo quando diferentes modalidades de cirurgia são comparadas. ${ }^{30}$

\section{Gravidez}

A cirurgia bariátrica melhora a fertilidade feminina. ${ }^{29,31}$ Entretanto, potencialmente agrava a taxa de deficiência de ferro em mulheres em idade reprodutiva, especialmente porque o consumo de ferro aumenta na gestação. Anemia não é um risco apenas para a mãe, mas também para o bebê. Dados da literatura sugerem que mães com deficiência de ferro têm maior risco de parto prematuro e recém-nascido de baixo peso. ${ }^{32}$ Suplemento de ferro oral associado a vitaminas, folato e vitamina $B_{12}$ é importante durante a gestação. Gurewitsch e colaboradores sugeriram que mulheres em idade fértil submetidas à cirurgia de bypass gástrico deveriam receber tratamento com ferro parenteral pré-operatório ou préconcepção para evitar os riscos de transfusão durante a gestação. ${ }^{33}$ Procedimentos de banda, que preservam a absorção de ferro no duodeno, podem também ser uma melhor opção que bypass gástrico para algumas mulheres que planejam ter filhos após a cirurgia.

\section{Adolescência}

Cirurgiões estão realizando bypass gástrico e procedimentos de banda em adolescentes com baixa mortalidade e melhora na qualidade de vida. ${ }^{34} \mathrm{Um}$ grande inconveniente para os cirurgiões, entretanto, é que isto pode resultar em deficiências nutricionais prolongadas, incluindo deficiência de ferro. Em adição ao rápido crescimento, os mecanismos propostos para a deficiência de ferro em adolescentes incluem dieta pobre em ferro e perda menstrual. ${ }^{35,36}$ 


\section{Repercussões clínicas e abordagem diagnóstica}

Clinicamente, os pacientes com deficiência de ferro e anemia ferropriva apresentam sintomas insidiosos relacionados à anemia, incluindo palidez, cansaço, adinamia, cefaleia, tonturas, dispneia e baixo desempenho no trabalho. Os exames laboratoriais de hemograma e avaliação dos estoques de ferro apresentam-se alterados. $\mathrm{O}$ esfregaço de sangue periférico indica hipocromia e microcitose. Entretanto, nas fases iniciais da deficiência de ferro, as hemáceas podem ser normocíticas e normocrômicas. Quando existe carência associada de folatos ou vitamina $B_{12}$, aparecem hemácias microcíticas e macrocíticas no esfregaço e os índices podem ser normais. O ferro sérico está diminuído (abaixo de $70 \mu \mathrm{g} / \mathrm{dL}$ para homens e $50 \mu \mathrm{g} / \mathrm{dL}$ para mulheres) e o TIBC (capacidade de ligação do ferro total) normal ou aumentado, o que leva à diminuição da saturação da transferrina a níveis menores que $10 \%$. A ferritina sérica está reduzida.

É importante enfatizar que entre o estado normal de estoques de ferro e a anemia ferropriva existem situações intermediárias em que a morfologia das hemáceas (microcitose e hipocromia), o ferro sérico, o TIBC e a ferritina não apresentam ainda todas as alterações características da anemia ferropriva acima descritas. Assim, existe uma fase em que a eritropoese já está comprometida, existe anemia leve a moderada, mas as hemáceas ainda são normocíticas e normocrômicas. A ferritina sérica está diminuída, mas em níveis acima de $10 \mu \mathrm{g} / \mathrm{L}$. Outra situação intermediária caracteriza-se pela redução ou ausência de ferro nos depósitos, sem, entretanto, haver anemia. As hemáceas são normocíticas e normocrômicas, a ferritina está diminuída, mas os seus níveis situam-se entre 25 e $10 \mu \mathrm{g} / \mathrm{L} .^{37}$

\section{Prevenção}

Pacientes submetidos à ressecção gástrica e/ou intestinal, incluindo as cirurgias bariátricas, requerem seguimento cuidadoso de seus parâmetros hematológicos com contagem hematológica, volume corpuscular médio (VCM), hemoglobina corpuscular média das hemáceas (HCM), ferro sérico, ferritina e TIBC. Sugere-se que estes exames sejam avaliados no pré-operatório para que possíveis deficiências de ferro possam ser corrigidas antes do procedimento cirúrgico. Após a cirurgia, recomenda-se que estes valores devem ser monitorados semestralmente, na tentativa de prevenir deficiências nutricionais.

A maioria dos cirurgiões prescreve multivitamínicos para todos os pacientes, mas a quantidade de ferro fornecida em um comprimido de multivitamínicos é de aproximadamente $20 \mathrm{mg}$, sendo que estes não fornecem ferro suficiente e não previnem anemia ferropriva.$^{38} \mathrm{Um}$ estudo prospectivo duplo-cego comparou profilaxia com ferro versus placebo e demonstrou que pacientes recebendo placebo tiveram uma queda significativa nos níveis de ferritina dois anos após a cirurgia versus aqueles tratados com $320 \mathrm{mg}$ de ferro duas vezes ao dia. Portanto, a reposição de ferro por via oral demonstrou-se efetiva na prevenção e tratamento de anemia ferropriva em pacientes submetidos à cirurgia de bypass gástrtico. ${ }^{39}$ Apesar disso, anemia ferropriva sem resposta a ferro pode ser um problema nesta população, e a reposição de ferro no pré-operatório, associada à profilaxia de forma eficaz no pós-operatório, provavelmente exercem papel importante nesta população para a prevenção da anemia ferropriva.

É razoável iniciar a correção da anemia ferropriva em pacientes com níveis de hemoglobina $\geq 10 \mathrm{~g} / \mathrm{dL}$ com suplementação de ferro via oral. Desde que o ferro ferroso é mais rapidamente absorvido, recomenda-se o uso de sais ferrosos. Formulações de liberação contínua devem ser evitadas porque estas reduzem a quantidade de ferro que está presente para absorção no intestino delgado. ${ }^{13}$

\section{Tratamento}

Pacientes submetidos à cirurgia de ressecção gástrica e/ou intestinal absorvem menor quantidade de ferro diariamente se comparados com outros indivíduos com anemia ferropriva. Um comprimido de sulfato ferroso de $300 \mathrm{mg}$ fornece $40 \mathrm{mg}$ de ferro elementar. Pacientes devem ser orientados a ingerir um a dois comprimidos ( $40 \mathrm{mg}-80 \mathrm{mg}$ ) de ferro elementar por dia como profilaxia para prevenir deficiência de ferro, ou três a quatro comprimidos (120 mg$160 \mathrm{mg}$ ) por dia para tratamento de deficiência de ferro. Se os parâmetros de ferro não melhorarem dentro de vários meses de terapia, a dose deve ser aumentada. Em mulheres que apresentem ciclos menstruais, até seis comprimidos por dia de sulfato ferroso (240 mg de ferro elementar) podem ser necessários para corrigir a anemia ferropriva. ${ }^{13}$

Idealmente, o ferro oral deve ser tomado com o estômago vazio. Entretanto, isto pode aumentar os efeitos colaterais gastrintestinais, incluindo náusea, vômito, desconforto abdominal, cólicas abdominais e constipação nesses pacientes e, consequentemente, a adesão ao tratamento é baixa. A dose pode ser reduzida, mas o tempo necessário para repor os estoques de ferro (mínimo de quatro meses) será mais longo. Uma preparação líquida pode ser tentada quando os tabletes orais não forem tolerados. Evidências sugerem que algumas comidas alteram a absorção de ferro, e, se possível, não devem ser ingeridas com suplementos de ferro oral. Estes alimentos incluem chá, farelo, cereal e alimentos ricos em cálcio. ${ }^{16} \mathrm{~A}$ adição de vitamina $\mathrm{C}$ à suplementação de ferro oral pode prevenir e tratar a deficiência de ferro. A vitamina $\mathrm{C}$ aumenta a acidez do trato gastrintestinal de forma que facilita a conversão do ferro férrico para a forma ferrosa. ${ }^{40}$

Pacientes que se mantêm refratários à suplementação de ferro oral ou não são aderentes ao tratamento podem neces- 
sitar de reposição de ferro parenteral, especialmente se eles são sintomáticos ou se têm hemoglobina menor que $10 \mathrm{~g} / \mathrm{dL}$. Brolin e colaboradores observaram, em estudos prospectivos, que nenhum paciente com anemia severa (definido como hemoglobina $<10 \mathrm{~g} / \mathrm{dL}$ ) responderam ao tratamento com ferro oral isolado. ${ }^{41}$

Existem diferentes formulações para ferro parenteral, incluindo complexo de ferro dextram de baixo peso molecular e de alto peso molecular, sacarato de hidróxido de ferro e gluconato de ferro. ${ }^{37}$ Apesar de pouco frequente (38 eventos em 1 milhão de aplicações), todas as formulações de ferro parenteral estão associadas a efeitos colaterais de reação ao ferro livre caracterizada por uma lesão endotelial aguda e sintomas de náusea, hipotensão, taquicardia e dispneia. As formulações de ferro dextram estão associadas a reações anafiláticas, de forma que uma dose teste inicial deve ser inicialmente testada com supervisão médica. ${ }^{42,43} \mathrm{O}$ sacarato de hidróxido de ferro é disponível no Sistema Único de Saúde do Brasil e é a apresentação com menor taxa de efeitos colaterais. ${ }^{44}$

A dose total de ferro parenteral necessária para o tratamento da anemia ferropriva depende da intensidade da deficiência de ferro, do peso do paciente e da reserva necessária de ferro de cada indivíduo, sendo que uma fórmula para o cálculo da quantidade de ferro parenteral a ser reposto é disponível na bula do medicamento. Recomenda-se a infusão endovenosa de duas ampolas de sacarato de ferro, diluídas em soro físiológico, com um tempo mínimo de infusão de 15 minutos, duas a três vezes por semana, até se completar a reposição da quantidade necessária estimada. Uma ampola de $5 \mathrm{~mL}$ contém $100 \mathrm{mg}$ de ferro. A maioria dos pacientes necessita de cerca de 1 a 2 gramas de ferro para serem repostos.

Transfusões de sangue ou intervenções cirúrgicas como reversão do bypass gástrico podem ser necessárias em pacientes com anemia ferropriva refratária. No caso de mulheres que apresentem ciclos menstruais, a histerectomia pode ser considerada, dependendo da gravidade da anemia. ${ }^{13}$

\section{Associação de outras causas de anemia}

Correção da anemia por períodos prolongados permanece um problema. É importante excluir outras etiologias para uma anemia persistente. Alguns estudos mostram que até $50 \%$ dos casos de anemia em pacientes gastrectomizados podem não ser atribuídos somente à deficiência de ferro. ${ }^{41} \mathrm{Um}$ estudo prospectivo, duplo cego, testando a eficácia de profilaxia com ferro oral nesses pacientes observou que vitaminas e ferro oral foram efetivos para corrigir a deficiência de ferro, mas não foram eficazes para evitar a ocorrência de anemia por outras causas. As demais causas de anemia nesse grupo de pacientes incluem doença crônica, doenças endocrinológicas, deficiência de folato e vitamina $\mathrm{B}_{12} \cdot 17,27,41,45$

\section{Conclusões}

A deficiência de ferro e anemia ferropriva são complicações frequentemente observadas em pacientes gastrectomizados, sobretudo naqueles submetidos a cirurgias bariátricas. A reposição de ferro de forma eficaz e precoce pode evitar essa complicação. No entanto, casos mais graves e refratários podem ser encontrados devido à complexidade das causas de deficiência de ferro nesses pacientes. A avaliação para essa condição deve ser feita de forma eficaz e sistemática para a determinação da melhor forma terapêutica e da presença de outras causas de anemia.

\begin{abstract}
Iron deficiency anemia is associated with gastrectomy used in the treatment of peptic ulcers and gastric cancer, and also in bariatric surgery for the treatment of obesity. Bariatric surgery involves restrictive and bypass procedures, gastrectomy and small intestine resection. There are several reasons why iron deficiency occurs in these patients including: (1) diminished iron intake due to meat intolerance (2) diminished gastric acid secretion due to loss of parietal cells which impairs the solubilization of ferric iron to form ferrous iron and (3) exclusion of the duodenum with consequent elimination of the main site of iron absorption and restriction in the action of pancreatic enzymes to release iron bound to the heme. Early clinical evaluation and use of iron supplements may be effective to prevent iron deficiency anemia in this population. Patients who remain refractory to oral supplementation may require parenteral iron administration. All patients require periodical and lifelong follow-up of hematological and iron parameters. Rev. Bras. Hematol. Hemoter. 2010;32(Supl.2):78-83.
\end{abstract}

Key words: Anemia, iron-deficiency; gastrectomy; bariatric surgery.

\section{Referências Bibliográficas}

1. Nunobe S, Okaro A, Sasako M, Saka M, Fukagawa T, Katai H, et al. Billroth 1 versus Roux-en-Y reconstructions: a quality-of-life survey at 5 years. Int J Clin Oncol. 2007;12(6):433-9.

2. Bult MJ, van Dalen T, Muller AF. Surgical treatment of obesity. Eur J Endocrinol. 2008;158(2):135-45.

3. Bessesen DH. Update on obesity. J Clin Endocrinol Metab. 2008; 93(6):2027-34.

4. Schweitzer DH, Posthuma EF. Prevention of vitamin and mineral deficiencies after bariatric surgery: evidence and algorithms. Obes Surg. 2008;18(11):1485-8.

5. Santos LM, Oliveira IV, Peters LR, Conde WL. Trends in morbid obesity and in bariatric surgeries covered by the Brazilian Public Health System. Obes Surg. 2008 Jun 7. [Epub ahead of print]

6. Salameh JR. Bariatric surgery: past and present. Am J Med Sci. 2006;331(4):194-200.

7. Hocking MP, Duerson MC, OLeary JP, Woodward ER. Jejunoileal bypass for morbid obesity. Late follow-up in 100 cases. N Engl J Med. 1983;308(17):995-9.

8. Sjöström L, Narbro K, Sjöström CD, Karason K, Larsson B, Wedel $\mathrm{H}$, et al. Effects of bariatric surgery on mortality in Swedish obese subjects. N Engl J Med. 2007;357(8):741-52. 
9. Capella RF, Capella JF, Mandec H, Nath P. Vertical Banded Gastroplasty-Gastric Bypass: preliminary report. Obes Surg. 1991; 1(4):389-95.

10. Fobi MA. Surgical treatment of obesity: a review. J Natl Med Assoc. 2004;96(1):61-75.

11. Bloomberg RD, Fleishman A, Nalle JE, Herron DM, Kini S. Nutritional deficiencies following bariatric surgery: what have we learned? Obes Surg. 2005;15(2):145-54.

12. Vargas-Ruiz AG, Hernandez-Rivera G, Herrera MF. Prevalence of iron, folate, and vitamin B12 deficiency anemia after laparoscopic Roux-en-Y gastric bypass. Obes Surg. 2008;18(3):288-93.

13. Love AL, Billett HH. Obesity, bariatric surgery, and iron deficiency: true, true, true and related. Am J Hematol. 2008;83(5):403-9.

14. Kushner RF, Shanta Retelny V. Emergence of pica (ingestion of non-food substances) accompanying iron deficiency anemia after gastric bypass surgery. Obes Surg. 2005;15(10):1491-5.

15. Schweiger C, Weiss R, Berry E, Keidar A. Nutritional deficiencies in bariatric surgery candidates. Obes Surg. 2010;20(2):193-7

16. Conrad ME, Umbreit JN. Iron absorption and transport-an update. Am J Hematol. 2000;64(4):287-98.

17. Crowley LV, Seay J, Mullin G. Late effects of gastric bypass for obesity. Am J Gastroenterol. 1984;79(11):850-60.

18. Ernst B, Thurnheer M, Wilms B, Schultes B. Differential changes in dietary habits after gastric bypass versus gastric banding operations. Obes Surg. 2009;19(3):274-80.

19. Avinoah E, Ovnat A, Charuzi I. Nutritional status seven years after Roux-en-Y gastric bypass surgery. Surgery. 1992;111(2):37-142.

20. Brolin RL, Robertson LB, Kenler HA, Cody RP. Weight loss and dietary intake after vertical banded gastroplasty and Roux-en-Y gastric bypass. Ann Surg. 1994;220(6):782-90.

21. Gasteyger C, Suter M, Calmes JM, Gaillard RC, Giusti V. Changes in body composition, metabolic profile and nutritional status 24 months after gastric banding. Obes Surg. 2006;16(3):243-50.

22. Sugerman HJ, Londrey GL, Kellum JM, Wolf L, Liszka T, Engle $\mathrm{KM}$, et al. Weight loss with vertical banded gastroplasty and Roux$\mathrm{Y}$ gastric bypass for morbid obesity with selective versus random assignment. Am J Surg. 1989;157(1):93-102.

23. Brolin RE, LaMarca LB, Kenler HA, Cody RP. Malabsorptive gastric bypass in patients with superobesity. J Gastrointest Surg. 2002;6(2):195-203.

24. Skroubis G, Anesidis S, Kehagias I, Mead N, Vagenas K, Kalfarentzos F. Roux-en-Y gastric bypass versus a variant of biliopancreatic diversion in a non-superobese population: prospective comparison of the efficacy and the incidence of metabolic deficiencies. Obes Surg. 2006;16(4):488-95.

25. Andrews NC. Forging a field: the golden age of iron biology. Blood. 2008;112(2):219-30.

26. Andrews NC. Understanding heme transport. N Engl J Med. 2005; $353(23): 2508-9$

27. Fujioka K. Follow-up of nutritional and metabolic problems after bariatric surgery. Diabetes Care. 2005;28(2):481-4.

28. Shah M, Simha V, Garg A. Review: long-term impact of bariatric surgery on body weight, comorbidities, and nutritional status. J Clin Endocrinol Metab. 2006;91(11):4223-31.

29. Deitel M, Stone E, Kassam HA, Wilk EJ, Sutherland DJ. Gynecologic-obstetric changes after loss of massive excess weight following bariatric surgery. J Am Coll Nutr. 1988;7(2):147-53.

30. Sugerman HJ, Starkey JV, Birkenhauer R. A randomized prospective trial of gastric bypass versus vertical banded gastroplasty for morbid obesity and their effects on sweets versus non-sweets eaters. Ann Surg. 1987;205(6):613-24.
31. Martin LF, Finigan KM, Nolan TE. Pregnancy after adjustable gastric banding. Obstet Gynecol. 2000;95(6 Pt 1):927-30.

32. Allen LH. Biological mechanisms that might underlie iron's effects on fetal growth and preterm birth. J Nutr. 2001;131(2S-2):581S-589S.

33. Gurewitsch ED, Smith-Levitin M, Mack J. Pregnancy following gastric bypass surgery for morbid obesity. Obstet Gynecol. 1996; 88(4 Pt 2):658-61

34. Inge TH, Garcia V, Daniels S, Langford L, Kirk S, Roehrig H, et al. A multidisciplinary approach to the adolescent bariatric surgical patient. J Pediatr Surg. 2004;39(3):442-7.

35. Inge TH, Krebs NF, Garcia VF, Skelton JA, Guice KS, Strauss RS, et al. Bariatric surgery for severely overweight adolescents: concerns and recommendations. Pediatrics. 2004;114(1):217-23.

36. Nead KG, Halterman JS, Kaczorowski JM, Auinger P, Weitzman M. Overweight children and adolescents: a risk group for iron deficiency. Pediatrics 2004;114(1):104-8.

37. Cook JD. Diagnosis and management of iron-deficiency anaemia. Best Pract Res Clin Haematol. 2005;18(2):319-32.

38. Brolin RE, Gorman RC, Milgrim LM, Kenler HA. Multivitamin prophylaxis in prevention of post-gastric bypass vitamin and mineral deficiencies. Int J Obes. 1991;15(20):661-7.

39. Brolin RE, Gorman JH, Gorman RC, Petschenik AJ, Bradley LB, Kenler HA, et al. Prophylactic iron supplementation after Rouxen-Y gastric bypass: a prospective, double-blind, randomized study. Arch Surg. 1998;133(7):740-4.

40. Rhode BM, Shustik C, Christou NV, MacLean LD. Iron absorption and therapy after gastric bypass. Obes Surg. 1999;9(1):17-21.

41. Brolin RE, Gorman JH, Gorman RC, Petschenik AJ, Bradley LJ, Kenler HA, et al. Are vitamin B12 and folate deficiency clinically important after roux-en-Y gastric bypass? J Gastrointest Surg. 1998;2(5):436-42.

42. Chertow GM, Mason PD, Vaage-Nilsen O, Ahlmén J. Update on adverse drug events associated with parenteral iron. Nephrol Dial Transplant. 2006;21(2):378-82.

43. Muñoz M, Breymann C, García-Erce JA, Gómez-Ramírez S, Comin J, Bisbe E. Efficacy and safety of intravenous iron therapy as an alternative/adjunct to allogeneic blood transfusion. Vox Sang 2008; 94(3):172-83

44. Auerbach M, Ballard H, Glaspy J. Clinical update: intravenous iron for anaemia. Lancet. 2007;369(9572):1502-4.

45. Poitou Bernert C, Ciangura C, Coupaye M, Czernichow S, Bouillot JL, Basdevant A. Nutritional deficiency after gastric bypass: diagnosis, prevention and treatment. Diabetes Metab. 2007;33(1):13-24.

O tema foi sugerido e avaliado pelo coeditor deste fascículo educativo, Rodolfo Delfini Cançado, e pelo board interno da RBHH, e publicado após a concordância do editor, Milton Artur Ruiz.

Conflito de interesse: sem conflito de interesse

Recebido: 21/12/2009

Aceito: 16/01/2010 